\title{
APLICACIÓN DE TÉCNICAS DE MINERÍA DE TEXTOS SOBRE RESPUESTAS DE EXÁMENES EN FORMATO DE TEXTO LIBRE
}

\author{
Analía Guzmán, Martín Gustavo Casatti, María Alejandra Paz Menvielle, \\ Calixto Maldonado, Karina Ligorria y Nicolás Horenstein \\ CIDS - Centro de Investigación, Desarrollo y Transferencia en Sistemas de Información \\ Universidad Tecnológica Nacional, Facultad Regional Córdoba, Argentina \\ Maestro Marcelo Lopez esq. Cruz Roja Argentina
}

\section{RESUMEN}

Se presenta un análisis preliminar, utilizando técnicas de minería de textos, de las respuestas en formato de texto libre a exámenes por parte de estudiantes de la materia Paradigmas de Programación, de la carrera de Ingeniería en Sistemas de Información, dictada en la Universidad Tecnológica Nacional, Facultad Regional Córdoba. El trabajo presenta el desarrollo de las actividades de toma de muestras, preprocesamiento y análisis de minería de textos, exponiendo algunos indicadores obtenidos, tales como análisis de frecuencia, análisis de tokens y diversidad léxica. Por último se presentan las conclusiones y algunas líneas de trabajo futuro.

\section{PALABRAS CLAVE}

Minería de Textos, Analítica de Textos, Evaluaciones en Texto Libre

\section{CONTEXTO}

El presente trabajo forma parte de la fase 2 del proyecto de investigación y desarrollo que ha sido homologado por la Secretaría de Investigación, Desarrollo y Posgrado de la Universidad Tecnológica Nacional, desarrollado en el ámbito del CIDS - Centro de Investigación, Desarrollo y Transferencia en Sistemas de In-formación, denominado "Análisis y detección de patrones en un grafo conceptual construido a partir de respuestas escritas en forma textual a preguntas sobre un tema específico - Fase 2", el cual utiliza como caso testigo a la cátedra de Paradigmas de Programación, perteneciente a la carrera Ingeniería en Sistemas de Información, dictada en la Facultad Regional Córdoba, de la Universidad Tecnológica Nacional.

Como parte de dicho proyecto se diseñó y generó una base de conocimiento modelada como grafo dirigido, para gestionar las preguntas y respuestas de exámenes, escritas en forma textual, así como cualquier otro concepto contenido en el programa de estudios de la materia Paradigmas de Programación. Se construyó también, un prototipo para la gestión del conocimiento y la obtención de la calificación de las respuestas de los alumnos en los exámenes (Paz Menvielle M. y col., 2016; Paz Menvielle M. y col., 2017).

Posteriormente se fijó como objetivo utilizar la información que se graba en la base de conocimiento para la búsqueda, el análisis y la detección de patrones frecuentes en el grafo conceptual (Zhong J. y col. 2002; Hensman S. 2004), lo cual implicó continuar ampliando la infraestructura de la base y del prototipo para agregar información que permita almacenar el historial de las respuestas de los alumnos, junto con los indicadores de respuestas correctas e incorrectas, para buscar patrones relacionados a las respuestas de los alumnos en pertinencia y consistencia.

Se realizó una prueba de concepto con un tamaño de muestra de datos pequeño, considerando las posibles variantes que se pudieran presentar en un caso real y se comprobó que con el uso de algunas métricas descriptas (Hernández J. y Van Mieghem P., 2011) y su representación visual, se podían detectar patrones relacionados con el aprendizaje. 
Durante el transcurso del segundo semestre del año 2019, se obtuvo una muestra en base a las respuestas de los alumnos que estaban cursando en distintas comisiones por medio de un sistema prototipo para capturar respuestas de los estudiantes en archivos de texto, los cuales se utilizaron en la elaboración del presente trabajo.

Como parte del análisis inicial de este trabajo se efectuaron diferentes análisis usando herramientas de minería de textos (Kaur A. y Chopra D., 2016) para tener información que caracterice las respuestas y el uso de las palabras por parte de los alumnos. El objetivo final de esta etapa es poder comparar los resultados obtenidos con los que se esperan obtener una vez que los datos completos estén procesados y almacenados en la base de grafo y se realicen los análisis equivalentes.

\section{INTRODUCCION}

El reconocimiento automático, descripción, clasificación y agrupamiento de patrones son actividades importantes en una gran variedad de disciplinas científicas, como biología, psicología, medicina, inteligencia artificial, etc. Además es un campo de estudio muy utilizado actualmente ya que brinda la posibilidad de manejar grandes volúmenes de información, permitiendo detectar patrones que pueden ser relevantes y que de modo manual es muy difícil de conseguir (Pavlidis T., 2013).

Lo importante de detectar patrones en los datos es que se pueden inferir causas para la agrupación de los mismos y poder actuar en consecuencia si la situación lo requiere.

\subsection{Análisis de Patrones}

El análisis de patrones en el dominio bajo estudio, puede determinar si existen ciertos patrones que, aún, no siendo comunes en otras áreas, si lo son recurrentes en este dominio (You C., Holder L. y Cook D., 2008).

Dicho análisis puede servir para descubrir algunas características importantes que se relacionan con el aprendizaje, entre ellas:

- Los temas que revisten más dificultad de aprendizaje.

- La cantidad y tipos de errores más comunes y su relación con el tema o concepto evaluado.

- Las tendencias de los alumnos al momento de responder las mismas preguntas, es decir, si lo hacen con los mismos conceptos o, por el contrario, tienen una riqueza expresiva alta.

- Si las respuestas dadas por los alumnos tienen una correspondencia directa (literal) con respecto al material brindado para su estudio.

\subsection{Minería de Textos}

La minería de textos es una aplicación de la lingüística computacional y del procesamiento de textos que pretende facilitar la identificación y extracción de nuevo conocimiento a partir de colecciones de documentos o corpus textuales, los cuales se presentan como información no estructurada (Stavrianou A., Andritsos P. y Nicoloyannis N., 2007).

Inicialmente, la minería de textos debe facilitar el análisis de corpus textuales que a priori resultarían inmanejables debido a su tamaño. Así, un investigador podría analizar esos datos, identificar relaciones entre documentos y extraer conclusiones (Brun R. y Senso J., 2004).

Los procesos de minería de textos son los que nos permiten realizar la búsqueda de conocimiento sobre información no estructurada, tales como páginas web, documentos, artículos, etc. Existen diferentes niveles para tratar con el texto: desde el nivel de palabra a palabra, frase, documento, conjunto de documentos, conjunto de documentos con enlaces y aplicación. Cada nivel de procesamiento revelará diferente información sobre el texto.

1. Nivel de Palabra: Se puede procesar las palabras en base a diferentes criterios y propiedades, por ejemplo: propiedades (homónimos, sinónimos, hiponimia), Stop-Words, Stemming, N-Grams Frecuentes, relaciones Léxicas, entre otras. 
2. Nivel de Documento: Se puede procesar el texto de tres maneras fundamentales:

- $\quad$ Resumen (summarization).

- Visualización completa del documento.

- Segmentación de Textos.

En una etapa posterior se podrá contar con diferentes unidades temáticas y distintas muestras de estudiantes, lo que posibilitará realizar los análisis correspondientes entre distintos corpus de texto, entre ellos el análisis entre grupos de documentos o grupos de respuestas por cohorte.

\subsection{Procesamiento en Lenguaje $R$}

Para analizar la datos de las respuestas almacenadas en el archivo de texto generado por el prototipo auxiliar, se utilizará para el procesamiento y visualización gráfica el software R (Ihaka R., 1998).

$\mathrm{R}$ es un entorno y lenguaje de programación para la manipulación de datos, cálculo y representación gráfica, que cuenta con una serie de paquetes para la minería de textos, entre los que se encuentran: tm, wordcloud, ggplot 2 readr, dplyr, quanteda, entre otros.

En las próximas secciones se muestra la utilización de algunos paquetes para realizar el análisis de los datos.

\section{DESARROLLO}

El proyecto prevé analizar la información, almacenada en una base de datos de grafos, obtenida durante las instancias de evaluación en la que participan los estudiantes. Debido a que dicho almacenamiento se realizará por medio de un prototipo que aún se encuentra en etapa de desarrollo, para la elaboración del presente trabajo se utilizarán los archivos de texto intermedios que genera la herramienta de evaluación, los que constituyen la muestra bajo estudio.

\subsection{Recolección de las Muestras}

A fin de plantear los estudios comparativos se recolectaron dos tipos de mues-tras referentes a un capítulo de la materia, la primera relacionada a la información contenida en el material de estudio oficial de la cátedra y la segunda se obtuvo a partir de las respuestas de los alumnos que participaron en distintas instancias de exámenes, respondiendo a preguntas del capítulo elegido.

Para la segunda muestra se consideraron las respuestas de 100 estudiantes, los cuales respondieron a ocho preguntas cada uno, usando redacción de texto libre dando un total de 800 respuestas, lo que constituye el corpus bajo análisis, algunas de cuyas características se encuentran en la Tabla 1.

Tabla 1. Estadísticos de las muestras

\begin{tabular}{lll}
\hline documento & Palabras & Términos únicos \\
\hline APUNTEOBJETOS.TXT & 5880 & 1339 \\
RESPUESTAS.TXT & 13057 & 1634 \\
\hline
\end{tabular}

Para tomar las muestras se construyó un prototipo auxiliar para que los estudiantes utilicen, respondiendo a preguntas seleccionadas por los docentes y donde se almacenó la información resultante en archivos de textos, para su posterior procesamiento y grabación en una base de datos de grafos.

El equipo de investigación se encargó de cargar en el banco de preguntas del prototipo, el conjunto de preguntas confeccionadas por los docentes de la cátedra de Paradigmas de Programación y sus respuestas bases asociadas.

Para el ingreso del texto de las respuestas por arte de los estudiantes se dispuso de una interface gráfica de usuario (GUI) creada en el prototipo auxiliar, para permitir al estudiante escribir las respuestas a las preguntas solicitadas. 


\subsection{Carga de los Datos de las Muestras}

Para realizar la carga de datos en el entorno de trabajo de $\mathrm{R}$ (R-Studio) se utilizaron las funciones implementadas en la librería readtext, la cual incluye la posibilidad de utilizar diversas fuentes de datos (archivos de texto fijo, separados por comas, documentos PDF y Word, y hasta documentos descargados directamente desde direcciones URL).

Se cargó en una única estructura de datos los dos archivos de muestra disponible, el que corresponde a las respuestas de los estudiantes (RESPUESTAS.TXT) y el que corresponde al material de estudio de la materia (APUNTEOBJE-TOS.TXT) tal como se puede apreciar en el Listado 1.1.

$$
\begin{gathered}
\text { Listado 1.1: Carga de archivos de datos } \\
\text { documentos <- readtext ( c ("RESPUESTAS.TXT" , "APUNTEOBJETOS.TXT" ) ) }
\end{gathered}
$$

Una vez con los datos en las correspondientes estructuras de trabajo se procedió al preprocesamiento de los mismos.

\subsection{Preprocesamiento de Datos}

El preprocesamiento es el tercer paso, dentro de los enunciados por (Velero A., 2017), trabajo en el cual se define al proceso como:

“... [se procede a] pasar todo el texto a minúsculas, eliminar signos de puntuación y palabras vacías así como reducir dimensiones para facilitar el estudio posterior ... ",

\subsubsection{Depuración de los Textos}

Antes de poder comenzar los análisis propios del contenido de los textos se realizó una depuración de los mismos para eliminar todo tipo de información textual que no incorporara valor al análisis.

A tal fin se corrió un procedimiento que elimina el "ruido" de los archivos de texto, excluyendo todos los términos que no son relevantes en el análisis, como por ejemplo:

- Palabras de corte (stopword) del idioma español.

- Signos de puntuación, números y caracteres especiales.

- Cualquier otro contenido con una extensión menor o igual a los 3 caracteres.

Se utiliza, para este caso, n-gramas de longitud $>3$ ya que estadísticamente, para la lengua española, dichos n-gramas contienen mayor información semántica asociada a los conceptos bajo estudio (Teller V., 2000).

También se reemplazaron algunos plurales por su singular, se eliminaron caracteres acentuados y espacios duplicados y se transformó todo el texto a minúsculas.

\subsubsection{Obtención de los Corpus y Tokens}

Uno de los primeros pasos en cualquier análisis que se desee realizar sobre un texto, es la obtención del correspondiente "corpus" que representa las métricas del texto bajo análisis en cuanto a cantidad y frecuencia de palabras, de frases y de párrafos.

La obtención de los tokens (unidades textuales independientes) se puede realizar sobre el corpus de cada una de las muestras, para complementar los análisis y obtener indicadores específicos.

Ambos componentes (corpus y tokens) se obtienen a través de las funciones de la librería "quanteda" la cual incorpora diversos mecanismos de análisis cuantitativo de textos.

\subsection{Análisis de Text Mining}

Una vez realizadas las tareas previas de carga de datos y preprocesamiento tendientes a obtener un corpus consistente, en cada una de las muestras bajo análisis, se procedió a realizar análisis descriptivos de cada uno de los conjuntos de datos. 


\subsubsection{Análisis de Frecuencia}

Utilizando los mecanismos provistos por la librería "quanteda", específicamente la función textstat_frecuency() se obtuvieron dos tablas con las frecuencias absolutas y relativas de las palabras dentro del texto original, las mismas que se utilizaron para generar los correspondientes gráficos de frecuencia, tales como se pueden apreciar en la Figura 1.

Hay que mencionar que en el presente trabajo se presentan solamente las 10 frecuencias más altas, pero en el análisis general no se aplica ésta restricción.
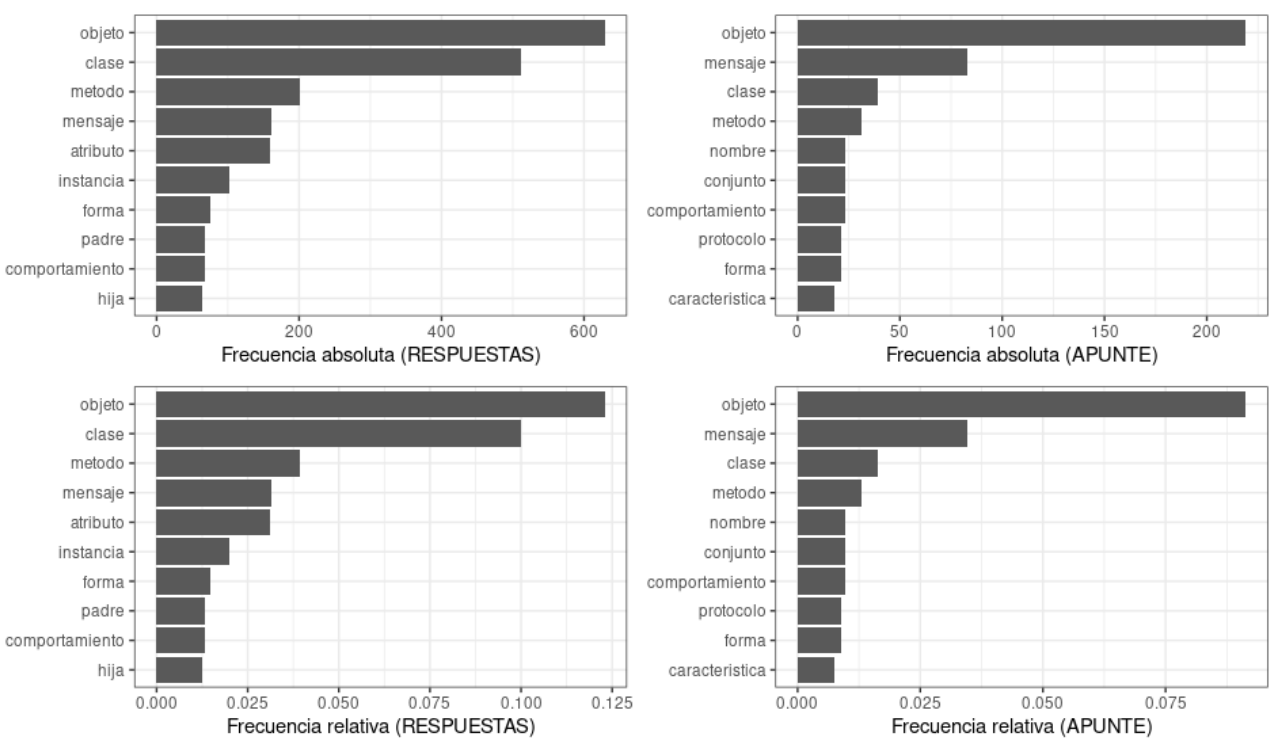

Figura 1. Frecuencias de términos (absolutas y relativas)

\subsubsection{Análisis de Tokens}

Los tokens, en un corpus de texto, son todos aquellos elementos que contienen información relevante con respecto al contenido. Por otra parte, existe un concepto relacionado a los tokens que es el de los tipos (types), siendo un tipo la expresión única de un token.

A modo de ejemplo, considerando un vocabulario compuesto por los dígitos 0 a 9 , se puede definir la secuencia representada por los dígitos 206520054. Dicha secuencia tiene 9 tokens, pero tiene solamente 5 types $(2,0,6,5,4)$.

Por medio de estos dos conceptos se puede realizar un análisis comparativo (token -> token y type -> type) entre corpus, y un análisis descriptivo (token $\rightarrow$ type) dentro de cada uno de los corpus. Estos indicadores individuales se calculan utilizando las funciones ntoken() y ntype() de R y los valores se pueden apreciar en la Tabla 2.

Tabla 2. Cantidad de tokens y types por documento

\begin{tabular}{lll}
\hline & APUNTE & RESPUESTAS \\
\hline TOKENS & 2398 & 5116 \\
TYPES & 965 & 942 \\
\hline
\end{tabular}

De los valores calculados, se puede apreciar que si bien hay una mayor cantidad de contenido en las respuestas de los estudiantes, eso no se traduce en una mayor expresividad, debido a que los types (tokens únicos) no acompañan ese incremento. El léxico utilizado es ligeramente superior en el caso del material de estudio (965 contra 942 types).

Un análisis interesante que se puede realizar, ahondando en estos conceptos, es el de la "diversidad léxica". 


\subsubsection{Análisis de Diversidad Léxica}

La diversidad léxica representa el vocabulario utilizado en contraste con la cantidad total de texto bajo análisis. Una diversidad léxica superior es indicativa de un mayor poder expresivo y de un dominio más amplio de la terminología.

La librería "quanteda" provee una función para calcular la diversidad léxica entre dos documentos (textstat_lexdiv()) la cual se utiliza para calcular y graficar el grado de diversidad entre los dos documentos de referencia.

Como se puede apreciar en la Figura 2 y en la Tabla 3 la diversidad léxica presente en el material de estudio prácticamente duplica la existente en las respuestas de los estudiantes.

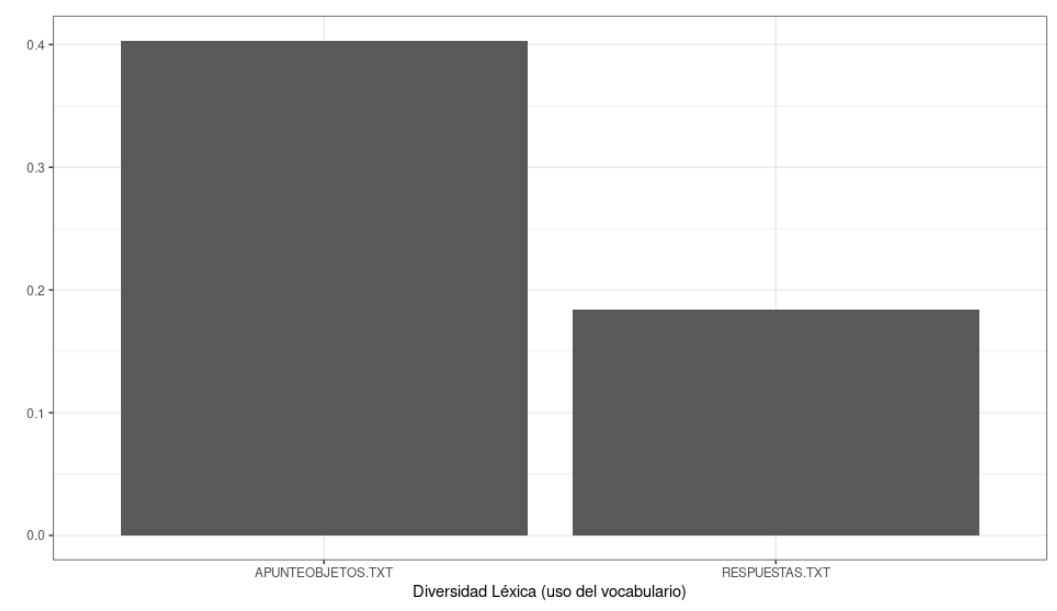

Figura 2. Diversidad léxica de los documentos

Tabla 3. Cálculo de la relación Token/Type

\begin{tabular}{ll}
\hline Documento & TTR \\
\hline APUNTEOBJETOS.TXT & 0.4030975 \\
RESPUESTAS.TXT & 0.1841642 \\
\hline
\end{tabular}

\section{CONCLUSIONES Y TRABAJOS FUTUROS}

Utilizando las técnicas de minería de textos a través del lenguaje R se posibilitó caracterizar ambas muestras, presentando algunos indicadores y métricas propias de cada una de ellas, así como también algunos análisis comparativos que permitieron observar coincidencias y discrepancias entre el contenido generado por los docentes y por los estudiantes de la misma cátedra.

Este trabajo sirve como referencia para el futuro análisis que se realice usando las mismas muestras recolectadas, pero almacenadas en una base de datos de grafos.

Se espera que dicha comparación permita confirmar la hipótesis de poder almacenar y recuperar la información almacenada en la base de datos de grafos para obtener patrones que sirvan en el proceso de enseñanza y aprendizaje.

Se estima que el aporte del proyecto brinde a la cátedra de Paradigmas de Programación la posibilidad de analizar el rendimiento de los alumnos en cuanto a la expresividad y al manejo del lenguaje, permitiendo ampliar la evaluación de las competencias. 


\section{REFERENCIAS}

Brun R. y Senso J., 2004, “Artıculo Minerı textual”. En: El profesional de la información 13.1, página 11.

Hensman, S., 2004, "Construction of conceptual graph representation of texts". En: Proceedings of the Student Research Workshop. HLT-NAACL. páginas 49-54.

Hernández J. y Van Mieghem P., 2011, "Classification of graph metrics". En: Delft University of Technology: Mekelweg, The Netherlands (2011), páginas 1-20.

Ihaka R., 1998, "R: Past and future history". En: Computing Science and Statistics 392396.

Kaur A. y Chopra D., 2016, "Comparison of text mining tools". En: 2016 5th International Conference on Reliability, Infocom Technologies and Optimization (Trends and Future Directions) (ICRITO). IEEE, páginas 186-192.

Pavlidis T., 2013. Structural pattern recognition. Volumen 1. Springer.

Paz Menvielle M. y col., 2016, "Arquitectura y operatoria de un sistema de corrección de exámenes automatizado, utilizando grafos dirigidos". En: IV Congreso Nacional de Ingeniería Informática y Sistemas de Información, CONAIISI, Universidad Católica de Salta, Facultad de Ingeniería, Argentina.

Paz Menvielle M. y col., 2017, "Modelo y herramienta de evaluación con la utilización de grafos como base de conocimiento para la corrección automatizada de exámenes en formato de texto". En: CLEI 2017: XLIII Conferencia Latinoamericana en Informática. Universidad Tecnológica Nacional Facultad Regional Córdoba, Argentina.

Stavrianou A., Andritsos P. y Nicoloyannis N., 2007, "Overview and semantic issues of text mining”. En: ACM Sigmod Record 36., páginas 23-34.

Teller V., 2000, "Speech and language processing: an introduction to natural language processing, computational linguistics, and speech recognition”. En: Computational Linguistics 26.4, páginas 638-641.

Velero A., 2017, "Técnicas estadísticas en Minerı de Textos". En: (2017, accedido Septiembre 2020). url: https://idus.us.es/handle/11441/63197.

You C., Holder L. y Cook D., 2008, "Graph-based data mining in dynamic networks: Empirical comparison of compression-based and frequency-based subgraph mining”. En: 2008 IEEE International Conference on Data Mining Workshops. IEEE., páginas 929-938.

Zhong J. y col., 2002, “Conceptual graph matching for semantic search”. En: International Conference on Conceptual Structures. Springer, páginas 92-106. 